08

\title{
Широкополосные зеркала для спектрогелиографов солнечной обсерватории „КОРТЕС“
}

\author{
() С.А. Гарахин,, М.М. Барышева, ${ }^{1}$ Е.А. Вишняков, ${ }^{2}$ С.Ю. Зуев, ${ }^{1}$ А.С. Кириченко, ${ }^{2}$ С.В. Кузин, ${ }^{2}$ \\ В.Н. Полковников, ${ }^{1}$ Н.Н. Салащенко, ${ }^{1}$ М.В. Свечников, ${ }^{1}$ Н.И. Чхало ${ }^{1}$ \\ ${ }^{1}$ Институт фризики микроструктур РАН, \\ 607680 Нижний Новгород, Россия \\ ${ }^{2}$ Физический институт им. П.Н. Лебедева РАН, \\ 119991 Москва, Россия \\ e-mail: GarakhinS@yandex.ru
}

Поступило в Редакцию 14 апреля 2020 г.

В окончательной редакции 14 апреля 2020 г.

Принято к публикации 14 апреля 2020 г.

\begin{abstract}
Представлены результаты разработки, изготовления и анализа широкополосных $\mathrm{Mo} / \mathrm{Si}$ многослойных зеркал для спектрогелиографов солнечной обсерватории „КОРТЕС“, работающих в спектральных диапазонах 17-21 и 28-33 nm. Показаны преимущества стекового подхода. Приведены результаты измерений отражательных характеристик зеркал.
\end{abstract}

Ключевые слова: ЭУФ, спектрогелиограф, солнечная корона, широкополосные зеркала, апериодические зеркала, стековые структуры, рефлектометр с лазерно-плазменным источником.

DOI: 10.21883/JTF.2020.11.49978.133-20

\section{Введение}

Проектируемый эксперимент „Кортес“ представляет собой перспективную миссию по исследованию солнечной активности на борту Международной Космической Станции [1]. Научная аппаратура включает целый комплекс изображающих и спектральных приборов для проведения наблюдений в мягком рентгеновском и вакуумном ультрафиолетовом диапазонах практически всех слоев солнечной атмосферы: хромосферы, переходного слоя и короны. В частности, в состав аппаратуры входят бесщелевые спектрогелиографы, позволяющие одновременно получать пространственную и спектральную информацию в диапазонах 17-21 и 28-33 nm. Принцип действия спектрогелиографов заключается в следующем: излучение проходит через входной фильтр, попадает на дифракционную решетку, после чего фокусируется с помощью многослойного зеркала на ПЗС-детекторе (приборе с зарядовой связью), покрытом дополнительным фильтром (рис. 1).

В результате на кадре формируется серия монохроматических изображений Солнца, сжатых и сдвинутых относительно друг друга вдоль оси дисперсии. Данные спектрогелиографов позволяют восстанавливать дифференциальную меру эмиссии активных областей и солнечных вспышек [2]. Большой интерес также представляет возможность получения детальных спектров корональных выбросов массы.

Конкретные спектральные диапазоны 17-21 и $28-33$ nm выбраны с учетом научных задач. В частности, в диапазоне 17-21 nm находится много линий железа различной степени ионизации - FeXI, Fe XII, Fe XIII, Fe XXIV а также некоторые другие ионы [3]. На диапа- зон 28-33 nm приходится большее количество различных ионов, например Si VIII, IX, XI, Mg VIII, Ni XVIII, Ca XVIII, Fe XI, Fe XII, Fe XIII, Fe XIV, FeXV, Fe XVII, S XII, Не II и др. [3]. Перечисленные ионы формируются при различных условиях, соответствующих различным слоям солнечной атмосферы. Их регистрация позволяет проводить диагностики Солнца с разрешением по глубине.

Традиционной парой материалов, применяемой в диапазоне $12.5-35 \mathrm{~nm}$, является $\mathrm{Mo} / \mathrm{Si}$, что определяется гладкостью спектральных зависимостей показателей преломления Мо и $\mathrm{Si}$, относительно низким поглощением Si в этой области и подтвержденной высокой временной стабильностью отражательных характеристик таких структур. При этом периодические многослойные зеркала (ПМЗ), которые обеспечивают при фиксиро-

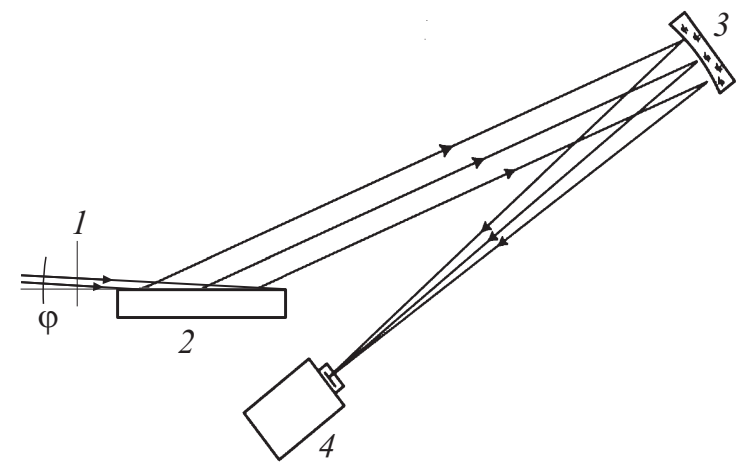

Рис. 1. Оптическая схема спектрогелиографов солнечной обсерватории „КОРТЕС“: 1 - защитный тонкопленочный фильтр, 2 - дифракционная решетка, 3 - многослойное рентгеновское зеркало, 4 - ПЗС-детектор. 

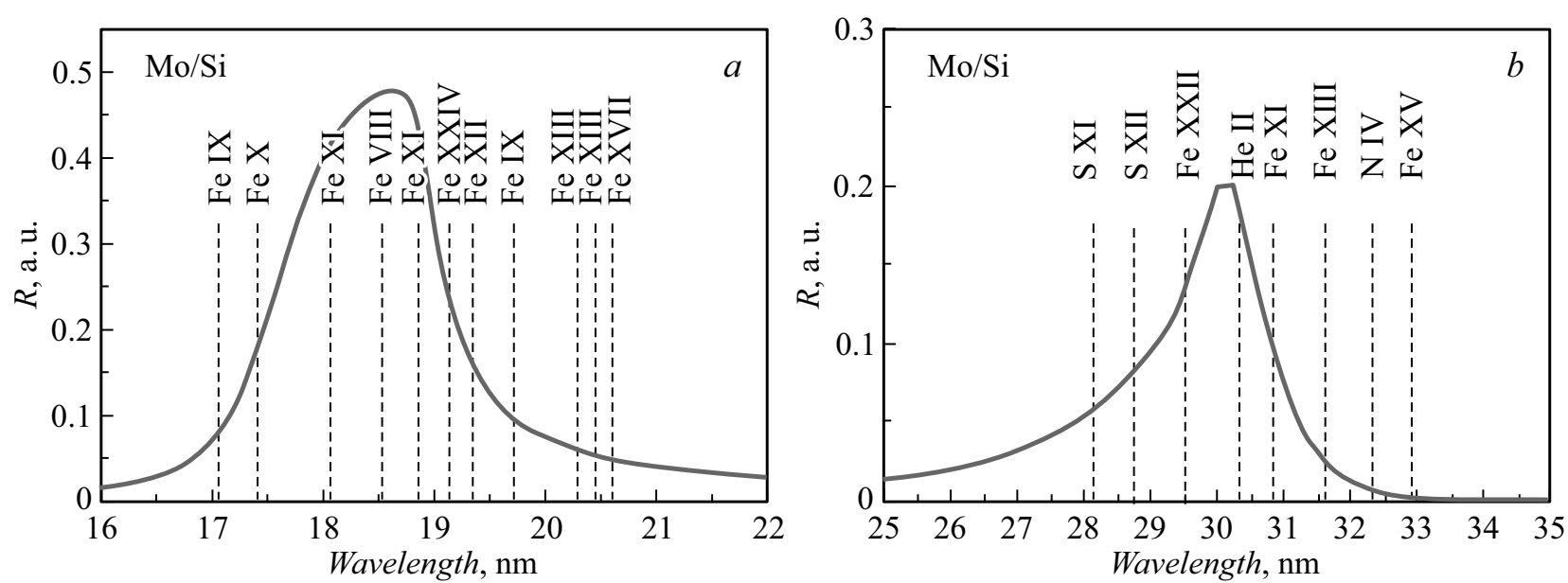

Рис. 2. Рабочие диапазоны спектрогелиографов „КОРТЕС“ и теоретические спектральные зависимости коэффициентов отражения соответствующих периодических рентгеновских зеркал Mo/Si: $a$ - для спектрального диапазона $17-21 \mathrm{~nm}, b-$ для спектрального диапазона $28-33 \mathrm{~nm}$.

ванном угле падения излучения достаточно высокий коэффициент отражения, имеющий вид резонансной кривой в относительно узком интервале длин волн, не могут эффективно использоваться для целей данных спектрогелиографов - нужен более широкий рабочий спектральный диапазон (рис. 2).

Таким образом, требуется разработать и синтезировать широкополосные зеркала, эффективно работающие в требуемых спектральных диапазонах.

\section{1. Оптимизация состава стековых структур}

В настоящий момент разработано два принципиально разных подхода к дизайну многослойных зеркал с уширенной кривой отражения. В обоих случаях за основу берется периодическое многослойное зеркало с максимумом пикового коэффициента в центральной области требуемого спектрального диапазона. Такое зеркало образовано тонкими чередующимися слоями материалов с разной диэлектрической проницаемостью. Далее толщины слоев такого зеркала математически оптимизируются таким образом, чтобы профиль коэффициента отражения соответствовал поставленной задаче.

В случае апериодического многослойного зеркала (АМЗ) все слои имеют индивидуальную толщину. Распределение толщин в жестком рентгеновском диапазоне подчиняется степенному закону (так называемые суперзеркала [4-6]) или хаотическому, рассчитываемому численно $[7,8]$ — в мягком рентгеновском и вакуумном ультрафиолетовом диапазонах. Этот подход осложняется тем, что плотности материалов в слоях нанометровой толщины зависят от толщины слоя. Например, плотность Мо увеличивается примерно от 0.77 до 0.97 от табличного значения при увеличении толщины слоя от $\sim 1.4$ до $5.5 \mathrm{~nm}$ [9]. Для снижения влияния этого эф- фекта, Кожевников и др. [9] усовершенствовали подход, в котором толщины всех слоев рассматриваются как параметры оптимизации. Критерий оптимизации модифицирован таким образом, чтобы уменьшить вариации толщины соседних одноименных слоев и тем самым сделать результат синтеза более предсказуемым. Это достигается ценой незначительного уменьшения интегрального коэффициента отражения и ухудшения его равномерности.

В работе [10] речь шла о расчете структуры $\mathrm{Mo} / \mathrm{Si}$ c равномерным отражением в диапазоне углов $0-18^{\circ}$ на длине волны $13.5 \mathrm{~nm}$. Однако несмотря на такую модификацию процесс синтеза АМЗ остается достаточно сложным: на начальной стадии напыления рост пленки происходит нелинейно, коэффициенты прилипания атомов конденсата отличаются, также происходит перемешивание материалов на границах между слоями. Технологические ошибки в толщинах слоя порядка $1-2 \%$, не оказывающие существенного влияния на коэффициент отражения ПМЗ, здесь вносят заметные искажения [8].

Второй подход так называемый стековый [11] (от английского stack) выглядит следующим образом. Многослойное зеркало представляет собой несколько напыленных одно на другое ПМЗ с разными характеристиками: $N_{i}$ - число стеков в широкополосной структуре, $d_{i}-$ период стековой структуры, $\gamma_{i}$ - доля сильно поглощающего слоя в периоде, где $i$ - номер периодического зеркала в составе стековой структуры. С увеличения числа ПМЗ и $N_{i}=1$ такая структура, очевидно, сводится к AM3. Отсюда же следует неизбежно меньшая гладкость кривой отражения оптимизированной стековой структуры и несколько меньшие коэффициенты отражения: при оптимизации состава АМЗ должен быть найден компромисс между гладкостью „плато“ и его высотой.

Достоинства стекового подхода определяются, главным образом, технологическим фактором. Фактически, чтобы корректно напылить $N$ различных толщин, необ- 


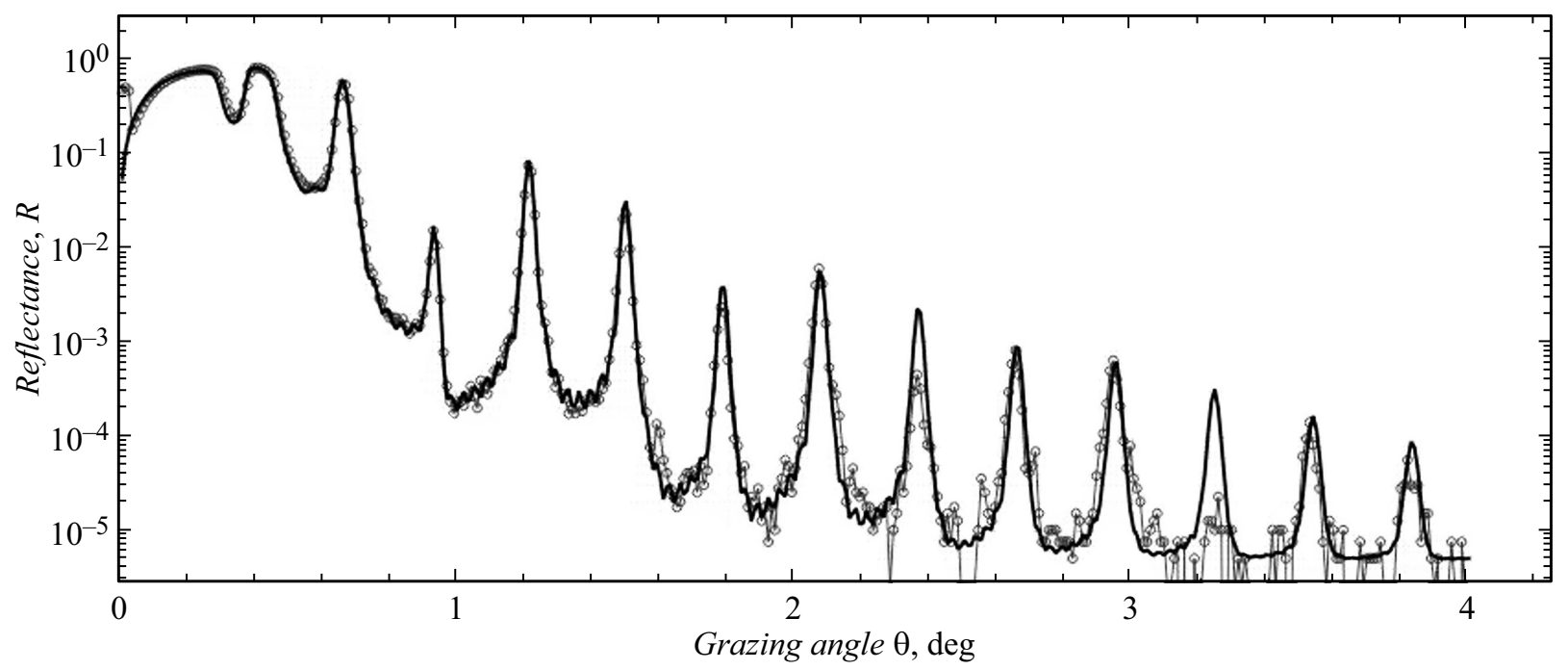

Рис. 3. Экспериментальная (точки) и теоретически подогнанная (сплошная кривая) угловые зависимости коэффициента отражения калибровочного ПМЗ, снятые на длине волны $0.154 \mathrm{~nm}$.

ходимо провести порядка $N$ калибровок параметров технологического процесса, что в случае АМЗ с большим (несколько десятков) числом слоев оказывается трудозатратным. Что касается решения обратной задачи, для классического АМЗ она практически нерешаема из-за большого числа параметров и неоднозначности полученного решения.

В случае стекового многослойного зеркала (СМЗ) число восстанавливаемых параметров существенно меньше, обратная задача может быть решена с высокой степенью точности, и проведена соответствующая коррекция технологического процесса. Приведенные соображения объясняют преимущество использования стекового дизайна при изготовлении широкополосных рентгеновских зеркал.

Вне зависимости от типа искомой структуры (апериодическая или стековая), математически задача оптимизации представляет собой минимизацию функционала

$$
F=\int\left[R(\lambda)-R^{\text {target }}\right]^{2} d \lambda
$$

где $R^{\text {target }}$ - целевая функция для коэффициента отражения определяет высоту „плато“. Интеграл вычисляется в области определения $R^{\text {target }}$ и рассматриваемая как функция толщин слоев. Процедура содержит несколько итераций: вначале высота плато выбирается на уровне, соответствующем периодическому зеркалу, и постепенно понижается до достижения удовлетворительной гладкости.

В случае AM3 параметрами в задаче оптимизации являются толщины слоев Мо и $\mathrm{Si}$ в парах $h_{i}(\mathrm{Mo}), h_{i}(\mathrm{Si})$, $i=1 \ldots N_{\mathrm{AMM}}$; общее число слоев $N_{\mathrm{AMM}}$ изначально берется равным таковому для периодической структуры и уменьшается в процессе оптимизации - отбрасываются глубинные слои, не оказывающее заметного влияния на расчетный коэффициент отражения. Таким образом, для AМ3 имеется всего несколько десятков параметров оптимизации.

Шероховатости и плотности для используемой пары материалов $\mathrm{Mo} / \mathrm{Si}$ известны [12], их зависимость от особенностей технологического процесса хорошо изучена на этапе изготовления ПМЗ. Оптимизация СМЗ в этой работе производилась при помощи алгоритма дифференциальной эволюции реализованного в рамках программы Multifitting [13]. В отличие от широко распространенного аналога IMD [14] Multifitting позволяет рассматривать число периодов в стеках $N_{i}$ как параметр подгона, что делает возможным решение задачи оптимизации в классе стековых структур. Другим важным преимуществом Multifitting является возможность одновременной подгонки нескольких кривых отражения, например, полученных в жестком и мягком рентгеновском диапазонах. Кроме того, переходные слои представляются в виде линейной комбинации простейших функций, включая функцию ошибок, наилучшим образом описывающую шероховатость и step-функцию, описывающую стехиометрические слои в переходной области, например, силицид молибдена в случае Mo/Si многослойного зеркала.

При вычислениях закладывались ширины переходных областей Мo-на-Si, равные $1.2 \mathrm{~nm}$ и $\mathrm{Si}-\mathrm{Ha}-\mathrm{Mo}-$ $0.6 \mathrm{~nm}[12]$, плотности пленок табличные.

В многопараметрических задачах всегда возникает вопрос об оптимальности найденного решения. Для повышения охвата параметрического пространства запускались серии автоматических подгонов со случайными начальными значениями искомых параметров, что увеличивало вероятность успеха. Тем не менее достижение глобального минимума остается негарантированным. В случае апериодических структур, удовлетворяющими заданному критерию, обычно оказываются несколько реализаций. На следующем этапе происходит отсев из 

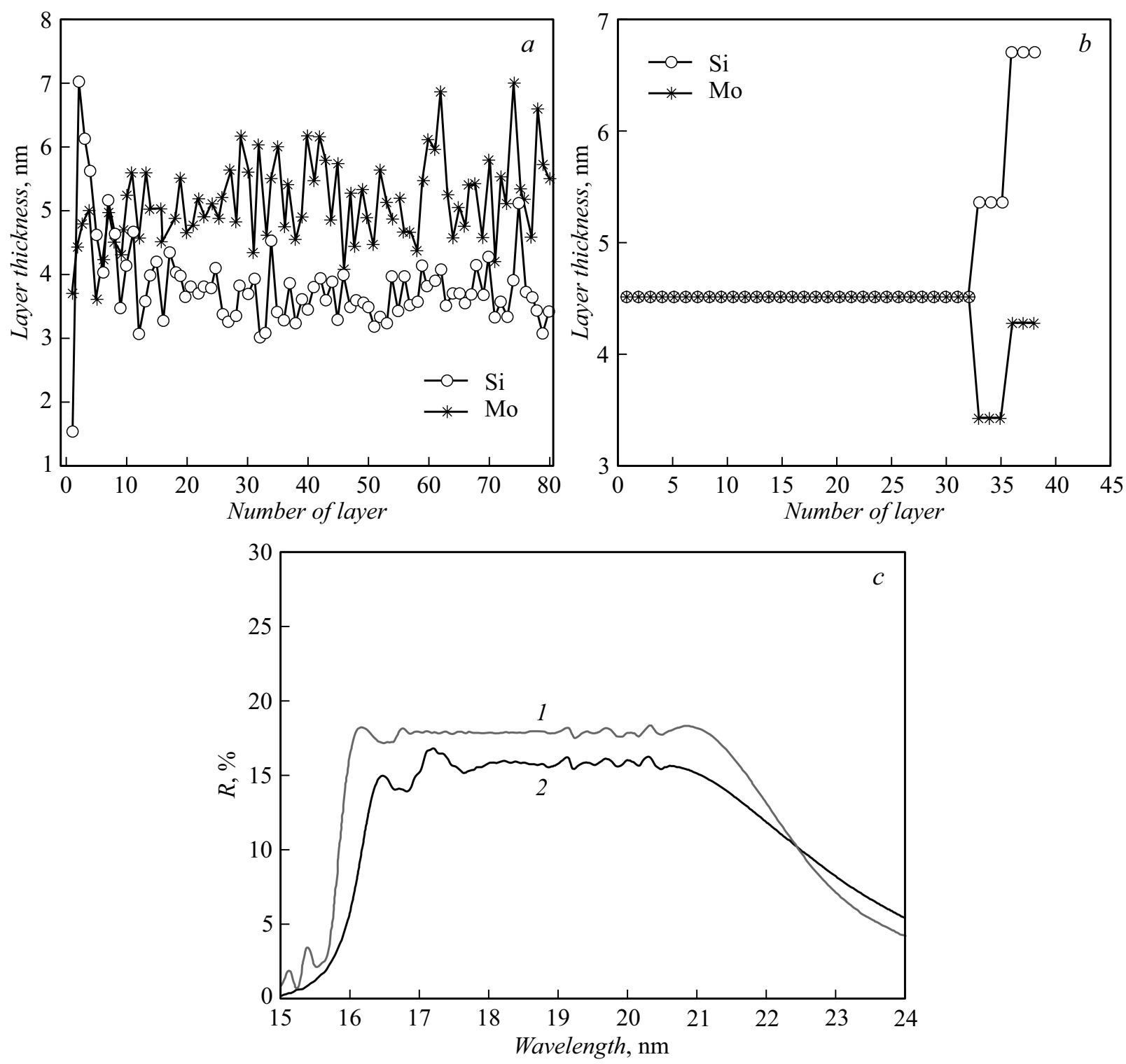

Рис. 4. Сравнение теоретических результатов для апериодического и стекового широкополосного зеркал с рабочим диапазоном 17-21 nm: зависимость толщины слоя от номера слоя для: $a$ - апериодической структуры и $b-$ стековой структуры (нумерация слоев начинается от подложки); $c$ - расчетные профили коэффициента отражения для апериодического $(1)$ и стекового (2) зеркала соответственно.

соображений устойчивости решения к малым возмущениям.

\section{2. Методика эксперимента}

Образцы изготавливались методом магнетронного напыления в атмосфере аргона при давлении $1 \cdot 10^{-3}$ Torr; в качестве подложек использовались кремниевые пластины для микроэлектронной промышленности с эффективной шероховатостью в диапазоне пространственных частот $0.024-65 \mu \mathrm{m}^{-1}, \sigma=0.3 \mathrm{~nm}$ [15]. Подробное описание технологической установки содержится в [16]. Толщина осажденной пленки материала определяется временем прохождения подложки над мишенью и величи- ной тока разряда, поэтому процессу изготовления зеркала предшествует процедура калибровки, которая состоит в синтезе нескольких предварительных периодических зеркал с толщинами слоев, соответствующими таковым в спроектированной широкополосной структуре. По данным малоугловой рентгеновской дифракции, полученным с использованием дифрактометра PANalitycal X'Pert Pro (длина волны $0.154 \mathrm{~nm}$ ), решается обратная задача, в результате которой с высокой точностью определяются параметры синтеза толщин слоев, содержащихся в требуемом широкополосном зеркале. Пример подобной калибровки (периодическое $\mathrm{Mo} / \mathrm{Si}$ зеркало с толщинами слоев, входящих в состав широкополосного зеркала) приведен на рис. 3. 

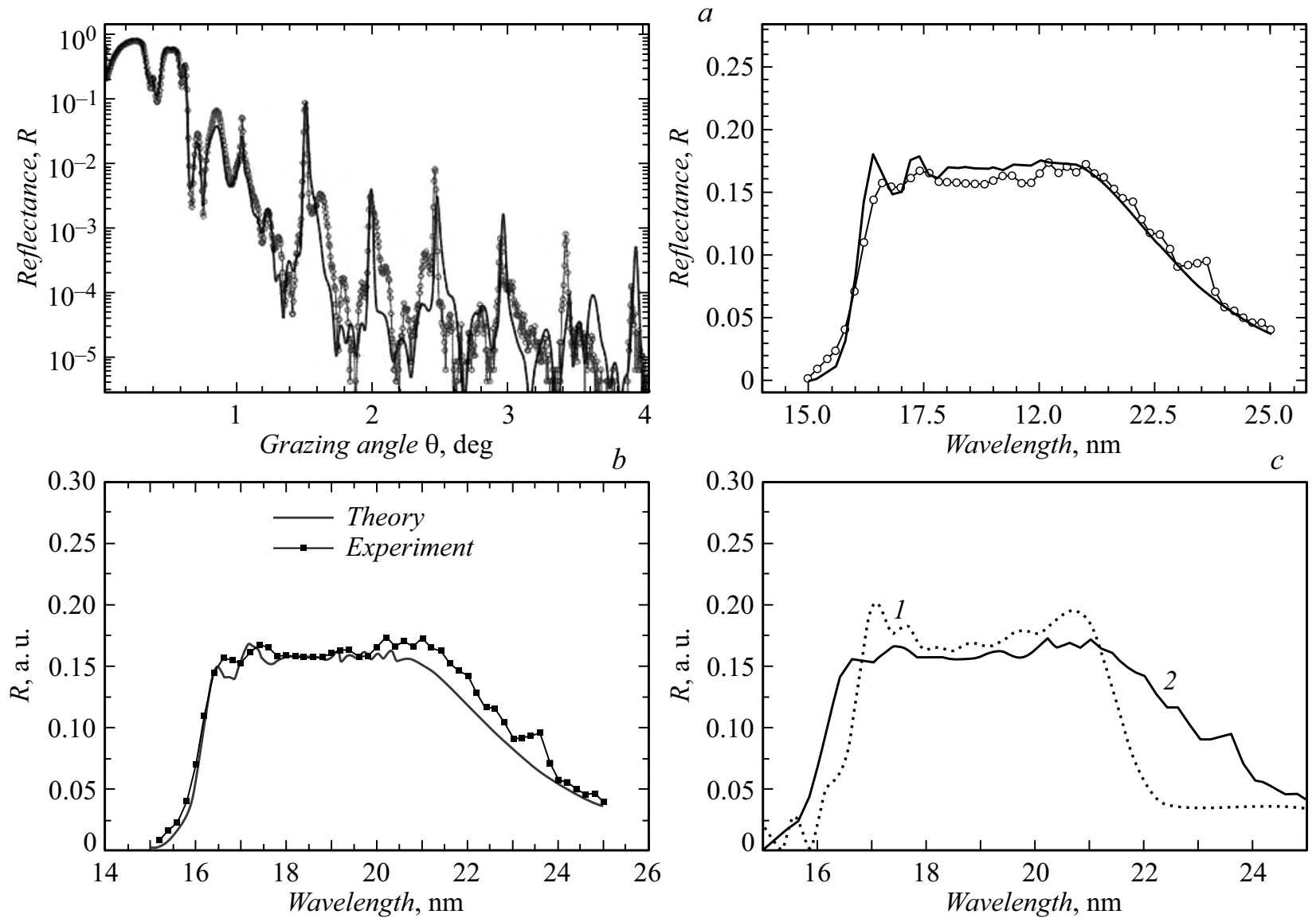

Рис. 5. $a$ - результаты фитинга отражательных характеристик стекового зеркала (точки - экспериментальные данные, линии фитинг); $b$ - сравнение с расчетным профилем; $c$ - сравнение полученного профиля стекового зеркала $(1)$ с профилем отражения синтезированного апериодического зеркала (2).

Однако неизбежный дрейф электрических параметров разряда, микропробои, незначительные изменения давления рабочего газа могут приводить к отклонению толщин пленок от заданных значений. Процедура изготовления многослойного покрытия, таким образом, итерационная, требующая определения параметров зеркала и соответствующей коррекции процесса синтеза.

В рабочих диапазонах длин волн $(17-21$ и $28-33 \mathrm{~nm})$ широкополосные зеркала изучаются на разработанном в ИФМ РАН рефлектометре, в котором монохроматизация излучения осуществляется с помощью высокоразрешающего спектрометра Черни-Тернера с плоской дифракционной решеткой и двумя сферическими коллимирующими зеркалами и лазерно-плазменным источником излучения [17].

На основе данных, полученных в жестком рентгеновском и вакуумном ультрафиолетовом диапазонах, решается обратная задача, для чего применяется авторская программа „Multifitting“, позволяющая одновременной производить подгонку нескольких кривых отражения, полученных в разных частотных диапазонах. В качестве параметров подгонки выступают характеристики стеков, составляющих широкополосное зеркало: толщи- ны и плотности материалов, шероховатости/масштабы переходных областей. В случае получения параметров восстановленной структуры, отличных от заданных, производится коррекция процесса напыления.

\section{3. Результаты и обсуждение}

В рамках настоящей работы были синтезированы и изучены образцы широкополосных Mo/Si CM3, изготовленных по описанной выше схеме и оптимизированных на равномерное отражение в диапазонах длин волн $17-21$ и $28-33 \mathrm{~nm}$.

\section{1. Широкополосное Mo/Si зеркало для спектрального диапазона $17-21 \mathrm{~nm}$}

Для стековой структуры дополнительными свободными параметрами являются величины $N_{i}$, число стеков было выбрано равным трем, сверху напылялась защитная пленка $\mathrm{Si}$, т.е. имеем всего 10 параметров, минимизирующих (1). В данном случае этого достаточно для удовлетворения заданным условиям, хотя, очевидно, с увеличением числа стеков теоретически можно полу- 

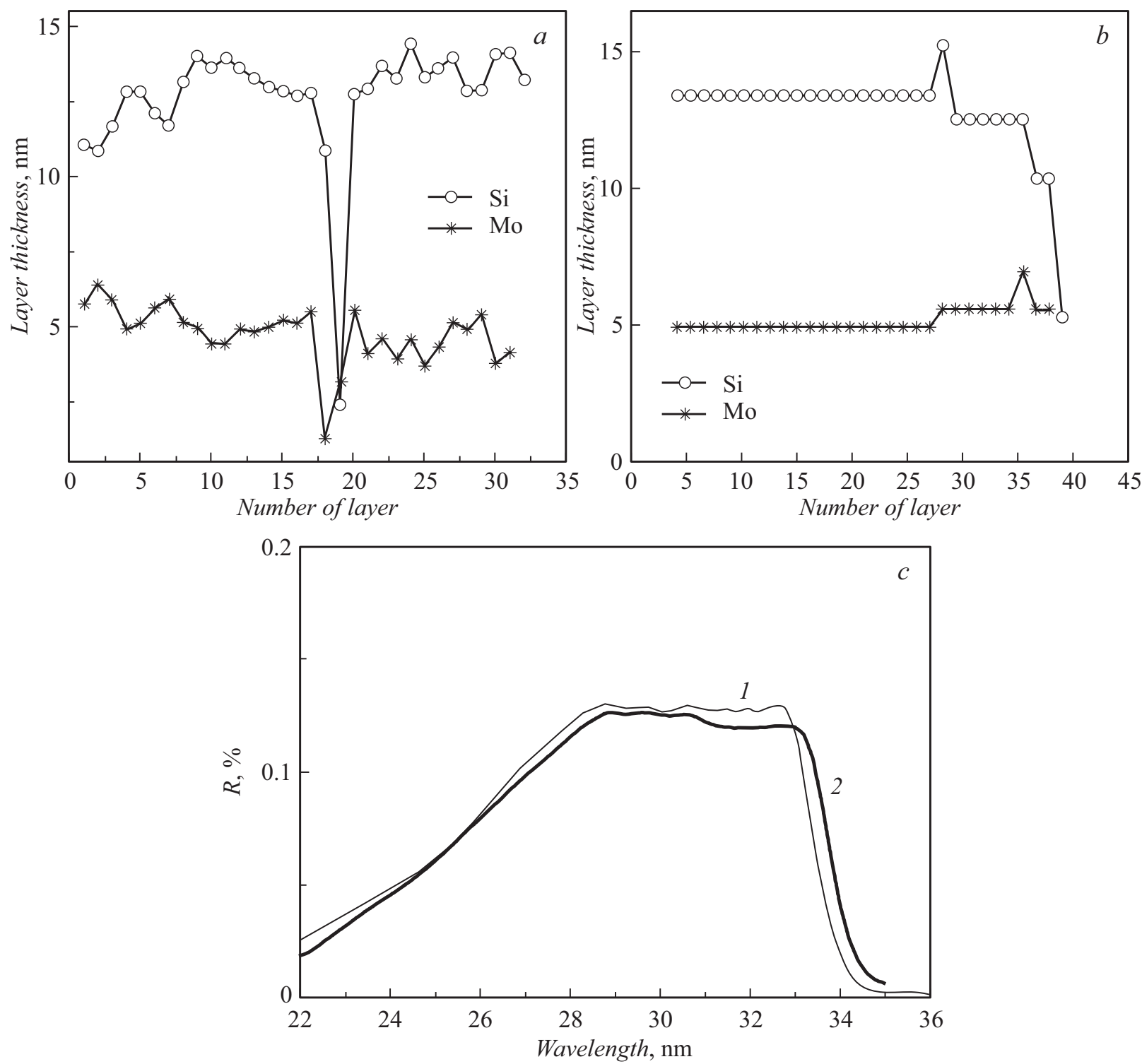

Рис. 6. Сравнение теоретических результатов для апериодического и стекового широкополосного зеркал с рабочим диапазоном $28-33 \mathrm{~nm}$ : зависимость толщины слоя от номера слоя для: $a-$ апериодической структуры, $b-$ стековой структуры (нумерация слоев начинается от подложки); $c$ - расчетные профили коэффициента отражения для апериодического $(1)$ и стекового (2) зеркала соответственно.

чать более совершенные кривые отражения (в пределе приходим к апериодической структуре).

Расчетный коэффициент отражения $R(\lambda)$ для зеркал $17-21 \mathrm{~nm}$ приведен на рис. 4. Можно видеть, что АМЗ превосходит СМЗ с точки зрения гладкости, средний коэффициент отражения в области $17-21 \mathrm{~nm}$ для него тоже несколько выше: $18 \%$ против $15.8 \%$. Однако, переходя к изготовлению многослойных апериодических структур, мы обнаруживаем, что это незначительное превосходство полностью нивелируется сложностью калибровки по толщинам и последующего синтеза. В то же время структуры „стекового“ типа позволяют обойтись всего несколькими предварительными итерациями синтеза.
Лучшая реализация напыления трехстекового $\mathrm{Mo} / \mathrm{Si}$ широкополосного зеркала для диапазона $17-21 \mathrm{~nm}$ проиллюстрирована на рис. 5. Последовательно проведенная процедура коррекции позволила за три итерации добиться „плато“ в области $17-21 \mathrm{~nm}$. Плотность Мо составила 0.98 от табличного значения, плотность $\mathrm{Si}-$ табличная. Значения шероховатостей для разных ПМЗ в составе стековой структуры составили $\sigma=0.5-0.9 \mathrm{~nm}$ (Mo-на-Si) и $\sigma=0.2-0.5 \mathrm{~nm}$ ( $\mathrm{Si}-$-на-Mo). Обращает на себя внимание чувствительность кривой отражения к ангстремным изменениям толщин пленок. В то же время незначительные отличия плотностей и значений шероховатости не приводят к заметному ухудшению вида кривой отражения, что важно с точки зрения решения 

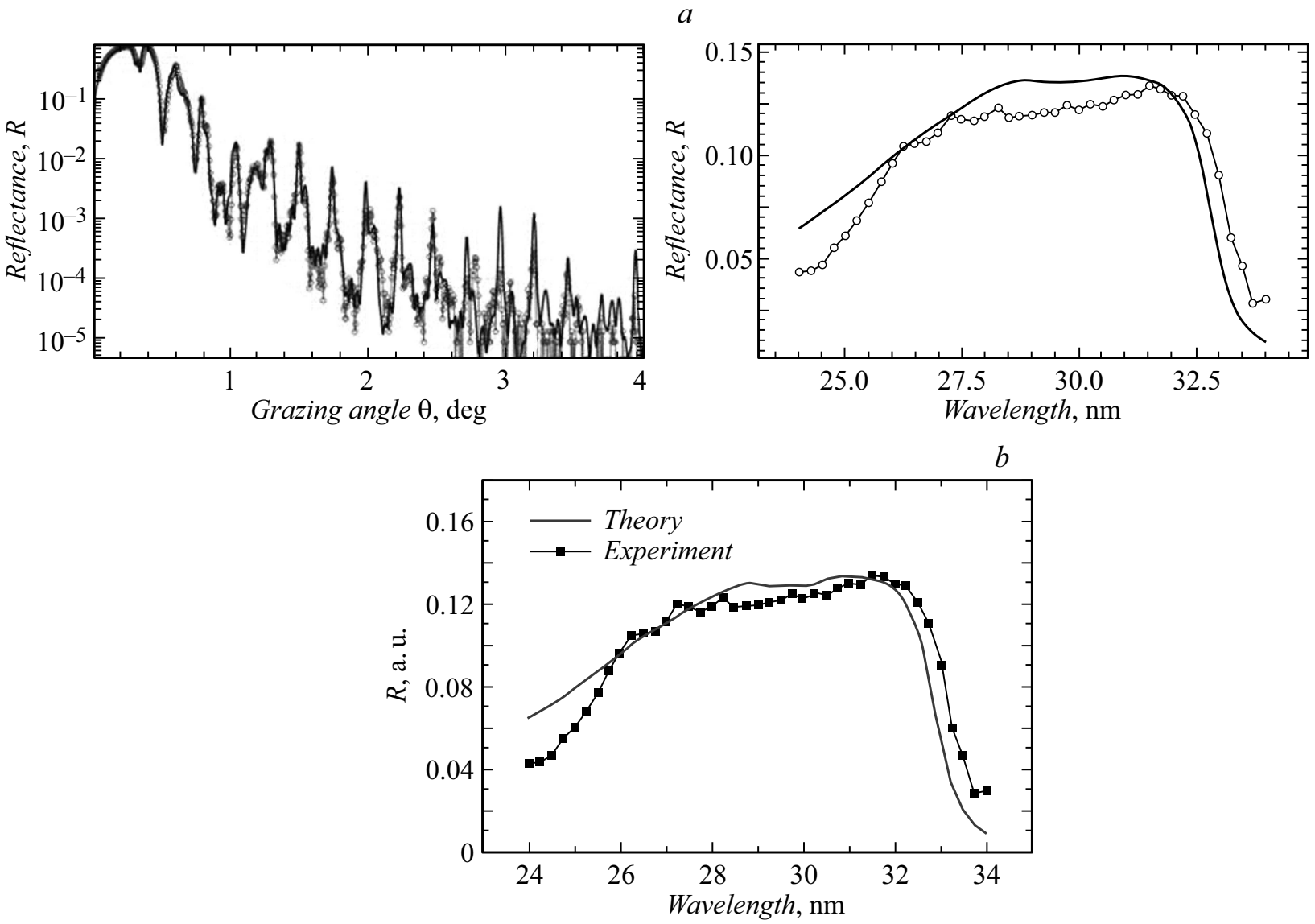

Рис. 7. $a$ - результаты фитинга отражательных характеристик стекового зеркала (точки - экспериментальные данные, линии фитинг); $b$ - сравнение с расчетным профилем.

прямой задачи, т.е. поиска оптимальных параметров CM3.

На рис. 5, а представлены результаты фитинга отражательных характеристик лучшей экспериментальной реализации стекового зеркала (точки - экспериментальные данные, линии - фитинг). Ниже приводится сравнение с расчетным профилем (рис. $5, b)$ и сравнение с профилем отражения синтезированного апериодического зеркала (рис. 5,c).

\section{2. Широкополосное Mo/Si зеркало для спектрального диапазона $28-3 \mathrm{~nm}$}

Для диапазона 28-33 nm было рассчитано теоретически и изготовлено $\mathrm{Mo} / \mathrm{Si} \mathrm{CM} 3$, образованное тремя ПМЗ с прослойками Мо и $\mathrm{Si}$ оптимальной толщины. Для удобства напыления эта структура представлена более мелким разбиением на 5 ПМЗ. Стековая структура практически не уступает по гладкости и величине коэффициента отражения апериодическому зеркалу, оптимизированному для этого диапазона.

При этом СМЗ так же, как для предыдущего спектрального диапазона, имеет более простой с точки зрения калибровки набор толщин слоев (рис. 6). Требуемой зависимости коэффициента отражения удалось достичь после четырех корректировок параметров синтеза. Спектральная характеристика экспериментального образца в сравнении с теоретическим профилем приведена на рис. 7, где также приведена подгонка кривых отражения в жестком и мягком рентгеновских диапазонах в программе Multifitting.

Плотности $\mathrm{Si}$, Мо аналогично СМЗ для $17-21 \mathrm{~nm}$ близки к табличным (толщины Мо находятся в диапазоне 5-10 nm, что существенно превышает характерную толщину кристаллизации для Мо), шероховатость также находится в рамках стандартных значений, наблюдается характерная для $\mathrm{Mo} / \mathrm{Si}$ асимметрия границ.

\section{Заключение}

Таким образом, разработана методика расчета, изготовления и анализа многослойных структур стекового типа. Созданы и изучены экспериментальные образцы $\mathrm{Mo} / \mathrm{Si}$ для спектрогелиографов солнечной обсерватории „КОРТЕС“. Для случая, когда была произведена последовательная корректировка технологических параметров, продемонстрировано хорошее совпадение теории и эксперимента. В течение нескольких коррекций задача была решена, что позволяет изготавливать широкопо- 
лосные зеркала стекового типа для различных приложений рентгеновской оптики.

\section{Финансирование работы}

Работа выполнена в рамках государственного задания № 0035-2014-0204 и при поддержке грантов РФФИ № 20-02-00708, 19-32-90154, и 18-07-00633, с использованием оборудования ЦКП „Физика и технологии микрои наноструктур“ при ИФМ РАН.

\section{Конфликт интересов}

Авторы заявляют, что у них нет конфликта интересов.

\section{Список литературы}

[1] Shestov S.V., Ulyanov A.S., Vishnyakov E.A., Pertsov A.A., Kuzin S.V. // Proceedings of the SPIE. 2014. Vol. 9144. P. $91443 \mathrm{G}$.

[2] Shestov S., Reva A., Kuzin S. // Astrophys. J. 2014. Vol. 780. N 1. P. 16.

[3] Slemzin V.A., Goryaev F.F., Kuzin S.V. // Plasma Phys. Rep. 2014. Vol. 40. N 11. P. 855-892.

[4] Вишняков Е.А., Каменеи, Ф.Ф., Кондратенко В.В., Лугинин М.С., Панченко А.В., Першин Ю.П., Пирожков А.С., Рагозин Е.Н. // Квант. электрон. 2012. Т. 42. Вып. 2. C. $143-152$.

[5] Joensen K.D., Voutov P., Szentgyorgyi A., Roll J., Gorenstein P., Hoghoj P., Christensenet F. // Appl. Opt. 1995. Vol. 34. N 34. P. 7935-7944.

[6] Kozhevnikov I.V., Bukreeva I.N., Ziegler E. // Nucl. Instrum. Methods Phys. Res. A. 2001. Vol. 460. N 2. P. 424.

[7] Бейгман И.Л., Пирожсков А.С., Рагозин Е.Н. // Письма в ЖЭТФ. 2001. Т. 74. Вып. 3. С. 167.

[8] Гайкович П.К., Полковников В.Н., Салащенко Н.Н., Чхало Н.И., Шеферс Ф., Соколов А. // Квант. электрон. Т. 46. Вып. 5. С. 406.

[9] Гарахин С.А., Мельчаков Е.Н., Полковников В.Н., Салащенко Н.Н., Чхало Н.И. // Квант. электрон. 2017. Т. 47. Вып. 4. С. 378-384.

[10] Kozhevnikov I.V., Yakshin A.E., Bijkerk F. // Optics Express. 2015. Vol. 23. N 7. P. 9276.

[11] Kuhlmann T., Yulin S., Feigl T., Kaiser N., Bernitzki H., Lauth H. // Proc. SPIE. 2002. Vol. 4688. P. 509.

[12] Braun S., Mai H., Mosset M., Scholz R., Leson A. // Jpn. J. Appl. Phys. 2002. Vol. 41. N 6B. P. 4074.

[13] Svechnikov M., Pariev D., Nechay A., Salashchenko N., Chkhalo N., Vainer Y., Gaman D. // J. Appl. Cryst. 2017. Vol. 50. P. 1428-1440.

[14] Windt D. // Computers in Phys. 1998. Vol. 12. N 4. P. 360.

[15] Барышева М.М., Вайнер Ю.А., Грибков Б.А., Зорина М.В., Пестов А.Е., Рогачев Д.Н., Салащенко Н.Н., Чхало Н.И. // Известия РАН. Сер. физическая. 2011. Т. 75. Вып. 1. С. 71.

[16] Andreev S.S., Akhsakhalyan A.D., Bibishkin M.A., Salashchenko N., Chkhalo N. // Centr. Europ. Journ. Phys. 2003. Vol. 1. P. 191-209.

[17] Гарахин С.А., Забродин И.Г., Зуев С.Ю., Каськов И.А., Лопатин А.Я., Нечай А.Н., Полковников В.Н., Салащенко Н.Н., Цыбин Н.Н., Чхало Н.И., Свечников М.В. // Квант. электрон. 2017. Т. 47. Вып. 4. С. 385. 